\title{
Durchdringende Schauer aus Blei und aus Kohle
}

\author{
Von Martin Deutschmann \\ Aus dem Max-Planck-Institut für Physik, Göttingen \\ (Z. Naturforschg. 9a, 477-488 [1954]; eingegangen am 21. Januar 1954)
}

\begin{abstract}
Es werden Messungen an 854 durchdringenden Schauern der kosmischen Strahlung beschrieben, die in Kohle und Blei in einer Nebelkammer ausgelöst wurden. Die mittlere Energie dieser Schauer liegt bei etwa $50 \mathrm{GeV}$. Durch Auszählen der Elektronenspuren wird der in $\pi^{0}$-Mesonen ausgestrahlte Teil der Schauerenergie bestimmt. Es ergibt sich ein integrales Energiespektrum, dessen Exponent $\gamma=1,5 \pm 0,2$ lautet. Die integrale Verteilung der Zahl der durchdringenden Teilchen, das Multiplizitätsspektrum, kann durch einen Exponenten $\delta=3,8 \pm 0,6$ beschrieben werden. Es wird geschlossen, daß die Zahl der durchdringenden Teilchen im Mittel mit der $\gamma / \delta=0,4$-ten Potenz $\_$der Primärenergie ansteigt. Die in Bleischauern umgesetzte Energie ist um das 1,9-fache höher als die der Kohleschauer. Die Zahl der durchdringenden Teilchen aus Blei und aus Kohle unterscheidet sich um 30\%; ein großer Unterschied besteht jedoch in der Zahl der stark ionisierenden Teilchen, diese werden aus Blei 5-mal häufiger emittiert als aus Kohle. Die Winkeldivergenz der Bleischauer ist um fast das Doppelte größer als die der Kohleschauer.
\end{abstract}

$\mathrm{A}$ us den Messungen an Mesonenschauern lassen sich Aussagen gewinnen über die Wechselwirkung der Nukleonen und $\pi$-Mesonen miteinander. Das bisher vorliegende experimentelle Material stammt allerdings zumeist aus Untersuchungen an Schauern aus schweren Elementen wie Blei oder Silber und Brom (Photoemulsion) ${ }^{1}$. Über leichte Elemente gibt es nicht so viele Messungen ${ }^{2-13}$, deren Ergebnisse zudem, was etwa die Wirkungsquerschnitte oder die mittleren Mesonenzahlen anbelangt, z.Tl. recht widerspruchsvoll sind. Dabei stellen gerade die Messungen an leichten Kernen eine mögliche Methode dar, etwas über den eigentlichen Grundprozeß, über die Mesonenentstehung beim Stoße zweier Nukleonen zu erfahren. Da die Messungen an reinem Wasserstoff ${ }^{9-13}$ wegen des niedrigen Wirkungsquerschnittes und wegen der bekannten technischen Schwierigkeiten so außerordentlich mühsam sind, erscheint es zweckmäßiger, genauere

1 Einen Überblick über solche Messungen enthält das Buch von W. Heisenberg, Vorträge über kosmische Strahlung, Springer Verlag, Berlin-GöttingenHeidelberg 1953.

2 G. Cocconi, Phys. Rev. 75, 1074 [1949].

${ }^{3}$ W. D. Walker, S. P. Walker u. K. Greisen, Phys. Rev. 80, 546 [1950].

${ }_{4}$ G. Salvini, Nuovo Cim. 8, 798 [1951].

${ }^{5}$ H. W. Boehmer u. M. S. Bridge, Phys. Rev. 85, 863 [1952].

${ }^{6}$ R. R. Brown, Phys. Rev. 87, 999 [1952].

7 I. R. Green, Phys. Rev. 80, 832 [1950].

${ }^{8}$ W. D. Walker, W. M. Duller u. I. D. Sorrels, Phys. Rev. 86, 865 [1952].

9 M. L. Vidale u. M. Schein, Nuovo Cim. 8, 774 [1951].

10 C. B. M. McCusker, N. A. Porter u. B. G. Wilson, Phys. Rev. 91, 384 [1953].
Untersuchungen an den besser zugänglichen leichten Elementen anzustellen. Um hier jedoch zu klareren Resultaten zu gelangen, die auch einen Vergleich mit den Theorien ${ }^{14-16}$ der Mesonenerzeugung zulassen, sollte man möglichst viele Eigenschaften der Schauer aus leichten und aus schweren Kernen gleichzeitig messen und dabei auch versuchen, die Energien der Schauer zu bestimmen, denn viele Meßgrößen, wie z. B. die Mesonenzahl, sind energieabhängig, und es ist möglich, daß mancheWidersprüche älterer Messungen auf die ungenügend berücksichtigten Schauerenergien zurückzuführen sind.

Messungen der Schauerenergien existieren bisher nur in recht geringer Zahl. So hat z. B. die BristolGruppe $^{17,18}$ die Energieabhängigkeit der Mesonenproduktion (zwischen etwa 1 und $10 \mathrm{GeV}$ ) in der Photoplatte bestimmt und hierzu die Winkelstreuung der Primärteilchen in der Emulsion beobachtet. Energien der Größenordnung $5 \cdot 10^{12} \mathrm{eV}$

11 H. Schultz, Dissertation Göttingen 1953.

12 A. B. Weaver, Phys. Rev. 90, 86 [1953].

${ }^{13}$ O. Kusumoto, S. Miyake, K. Suga u. Y. Watase, Phys. Rev. 90, 998 [1953].

${ }^{14}$ W. Heitler, Rev. Mod. Phys. 21, 113 [1949].

15 E. Fermi, Progr. Theor. Phys. 5, 570 [1951]; Phys. Rev. 81, 863 [1951].

${ }_{16}$ W. Heis en berg, Z. Phys. 126, 569 [1949], 133, 65 [1952]; Vorträge über kosmische Strahlung, 1. c. ${ }^{1}$.

${ }^{17}$ U. Camerini, J. H. Davies, P. H. Fowler, C. Franzinetti, M. Muirhead, W. O. Lock, D. H. Perkins u. G. Yekutieli, Phil. Mag. 42, 1241 [1951].

18 U. Camerini, W. O. Lock u. D. H. Perkins in ,Progress of Cosmic Ray Physics“, herausgegeben von J. G. Wilson, North-Holland Publishing Company, Amsterdam 1952. 
haben andererseits Kaplon und Mitarbb. ${ }^{19}$ aus der Winkelbreite der Schauer abgeleitet und auf diese Weise Aussagen über die mittleren Mesonenzahlen aus Blei, Messing und Photoemulsion gemacht. Um einheitliche Substanzen, insbesondere leichte Elemente, zu untersuchen, eignet sich die Nebelkammer sehr gut. Daher soll in vorliegender Arbeit über eine Nebelkammermessung an Blei und an Kohle berichtet werden, bei der zur Energiebestimmung die Elektronenkomponente herangezogen wurde, die aus dem Zerfall der $\pi^{0}$-Mesonen entsteht.

\section{Versuchsanordnung und Meßmethode}

Abb. 1 zeigt eine Skizze der Nebelkammer und der verwendeten Zählrohrsteuerung. Die Kammer von

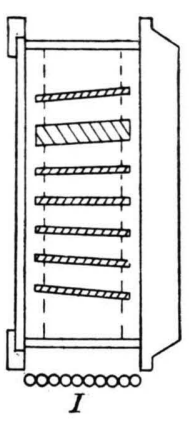

\section{$\widehat{10 \mathrm{~cm}}$} 프 B/ei Kohle

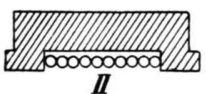

Abb. 1. Die Nebelkammer und die Zählrohranordnung. $74 \mathrm{~cm}$ Höhe und Breite und $20 \mathrm{~cm}$ beleuchteter Tiefe enthielt 6 Bleiplatten $\mathrm{zu}$ je $1,5 \mathrm{~cm}$ Dicke und eine Kohleplatte (Graphit), die $5 \mathrm{~cm}$ stark war; letztere befand sich zwischen der ersten und der zweiten Bleiplatte. Diese Anordnung erwies sich als günstig, denn sie erlaubte einmal zu entscheiden, ob die in der Kohle ausgelösten Schauer von durchdringenden Teilchen herrührten, und zum anderen bot die durch kein anderes Material unterbrochene Folge der 2. bis 6 . Bleiplatte die Möglichkeit, Elektronenkaskaden ohne Störung durch Übergangseffekte zu verfolgen. Daß die Abstände zwischen den einzelnen Platten relativ groß waren $(6,3 \mathrm{~cm})$, erleichterte das Auszählen der Elektronenspuren.

Gesteuert wurde die Kammer von den beiden Zählrohrlagen I und II zu je 10 Rohren (Durchmesser $3 \mathrm{~cm}$, Länge $60 \mathrm{~cm}$ in Lage I und $75 \mathrm{~cm}$ in Lage II), von denen mindestens drei pro Lage gleichzeitig ansprechen mußten. Lage II war durch $10 \mathrm{~cm}$ Blei von oben und von der Seite abgeschirmt, so daß nur Schauer mit mindestens drei Teilchen, die mehr als $10 \mathrm{~cm}$ Blei durchdrangen, die Expansion der Kammer auslösen konnten. Dadurch, daß beide Lagen sich unterhalb der Kammer befanden, war zwar ein Teil der schräg einfallenden Schauer in der Kammer nicht sichtbar, doch wurde dieser Ausfall in Kauf genommen, um Schauer mit ionisierenden wie neutralen Primärteilchen in ihrem wahren Häufigkeitsverhältnis zu erfassen. Mittels zweier Leicas, die mit 10,5 cm Schnei-

19 M. F. Kaplon, D. M. Ritson U. W. D. Walker, Phys. Rev. 90, 716 [1953].

der-Xenaren ausgerüstet waren, wurden im Abstand von $3,2 \mathrm{~m}$ ca. 10000 stereoskopische Aufnahmen gemacht. Die Kammer befand sich dabei auf der Zugspitze in $2960 \mathrm{~m}$ Höhe unter einem leichten Dach von durchschnittlich $6 \mathrm{~cm} \mathrm{Holz}+0,5 \mathrm{~mm}$ Eisen. Gegen Ende der Aufnahmezeit lag jedoch eine bis zu $1 \mathrm{~m}$ dicke Schneedecke auf einem Teil des Daches. So kamen die Primärteilchen von $1 / 5$ aller Schauer aus Schnee, doch zeigen die betreffenden Aufnahmen außer einem erhöhten Anteil an Begleitern des Primärteilchens keine Besonderheiten.

Aus der gesamten Aufnahmeserie wurden 854 Schauer* ausgesucht, welche innerhalb der Kammer entstanden und nicht von Luftschauern oder anderen dichteren Schauern begleitet waren. Außerdem wurde darauf geachtet, daß von den folgenden drei $\mathrm{Be}-$ dingungen mindestens eine erfüllt war: 1. Das Primärteilchen (gleichgültig ob ionisierend oder nicht) mußte eine oder mehrere Bleiplatten zuvor ohne Multiplikation durchdrungen haben. 2. Der Schauer mußte ein oder mehrere durchdringende Sekundärteilchen aufweisen. 3. Wenn die beiden ersten Bedingungen nicht erfüllt waren, so wurde darauf geachtet, daß vom Schauerursprung wenigstens eine stark ionisierende Spur ausging. Außerdem wurden von den schräg einfallenden Schauern nur diejenigen gezählt, die genügend lange im beleuchteten Kammerraum verblieben, d. h. es mußten alle Sekundärteilchen, die unter weniger als etwa $20^{\circ}$ zur Primärrichtung emittiert wurden, noch beim Durchgang durch die folgende Platte erkennbar sein. Schauer aus der untersten, der 6. Bleiplatte wurden überhaupt nicht gezählt, da bei ihnen zwischen durchdringenden und anderen Sekundärteilchen nicht zu unterscheiden war.

Beim Auszählen der durchdringenden Teilchen wurden folgende Fehlerquellen beachtet: In Schauern mit dichter Elektronenkomponente können einmal durchdringende Teilchen übersehen werden, und zum anderen können auch unzusammenhängende Elektronenspuren bei entsprechender Orientierung für solche durchdringender Teilchen gehalten werden. Ein ungefähres Maß für die Größe der Fehler erhält man durch unabhängiges Betrachten der gleichen Aufnahmen zu verschiedenen Zeiten. Das Ergebnis derartiger Proben waren mittlere Fehler von $15 \%$ im Einzelfall. Hinzu kommen zwar systematische Fehler durch Sekundärteilchen, die unter großem Winkel gegen die Schauerachse emittiert wurden, und deren Durchdringungsfähigkeit an den folgenden Platten nicht immer kontrolliert werden konnte. Die Beobachtung einer Reihe günstig orientierter Schauer zeigte jedoch, daß bei einem halben Öffnungswinkel von mindestens $20^{\circ} \mathrm{der}$ Fehler, der durch Weglassen aller stärker divergenten Spuren entsteht, im Mittel kleiner als $5 \%$ ist. Faßt man Schauer gleicher Größe zu Gruppen zusammen, so mitteln sich die ihrem Betrage nach überwiegenden zufälligen Fehler weitgehend heraus.

Die an $\pi^{0}$-Mesonen abgegebene Energie der Schauer wurde durch Auszählen der Elektronenkomponente

* Drei Schauer sind davon auf Tafel S. 484 a wiedergegeben (Abb. I-III). 


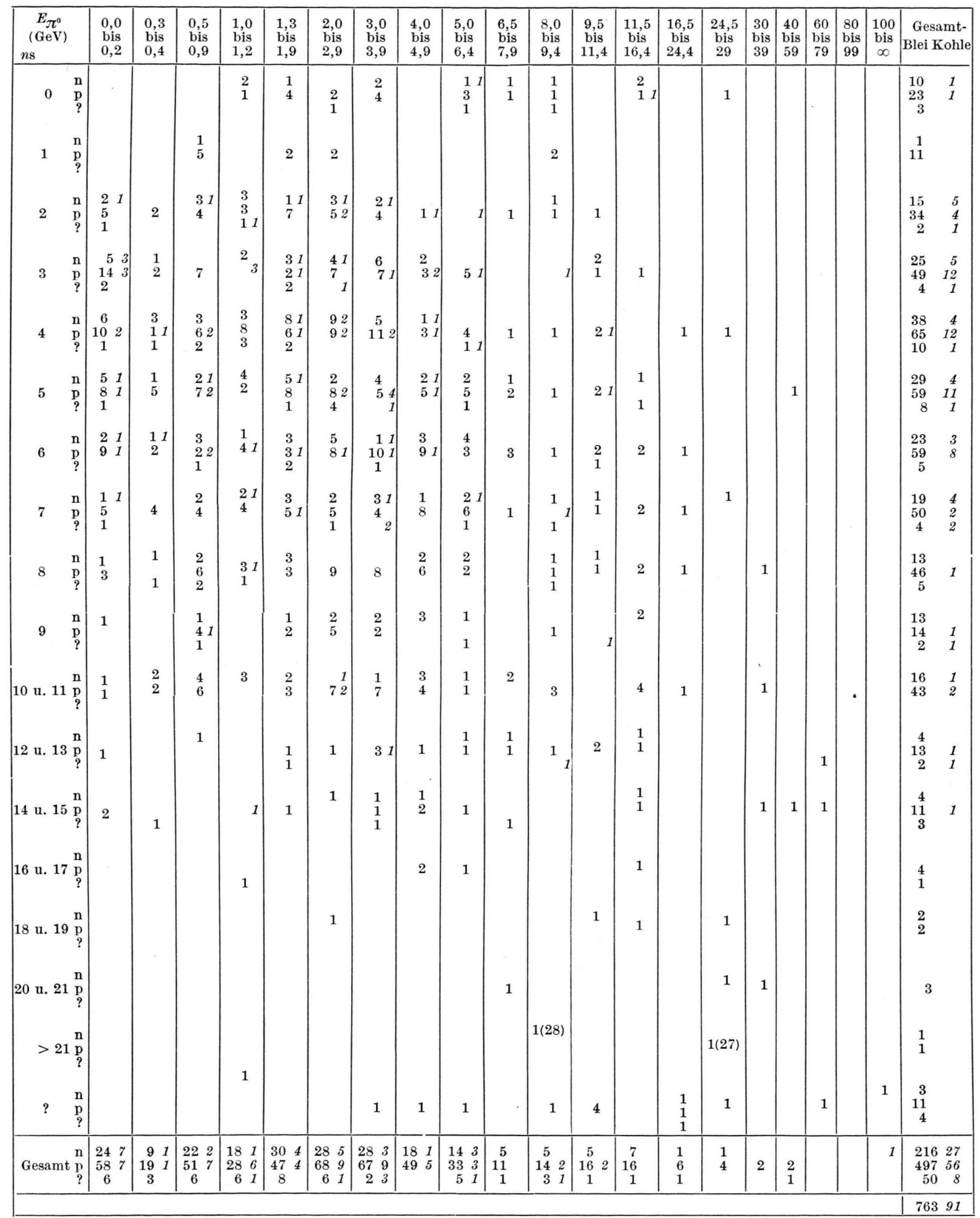

Tab. 1. Eine Aufstellung der 763 Blei- und der 91 Kohleschauer. Von links nach rechts steigt die Energie $E_{\pi^{0}}$ der Elektronen-Photonenkomponente der Schauer an, und von oben nach unten nimmt die Zahl $n_{\mathrm{s}}$ der durchdringenden Schauerteilchen zu. Die Zahl der Kohleschauer ist jeweils rechts von der der Bleischauer in kursiven Ziffern angegeben. 
unter möglichst vielen Bleiplatten bestimmt. Es wurde berücksichtigt, daß nicht, wie in der üblichen Fassung der Kaskadentheorie vorausgesetzt, ein einziges Elektron oder Photon die Kaskade startet, sondern, daß im allgemeinen mehrere $\pi^{0}$-Mesonen entstehen und in je zwei Photonen zerfallen. In einer demnächst erscheinenden Arbeit hat Ott** die Kaskadentheorie in diesem Sinne erweitert und die Resultate im Hinblick auf den praktischen Gebrauch in numerischer Form und als Kurven wiedergegeben. Nach diesen Kurven und nach den Erfahrungen bei der Auswertung der vorliegenden Aufnahmen zeigt es sich, daß der Fehler in der ermittelten Energie im wesentlichen durch die Schwankungen in der Kaskadenentwicklung und durch die Unsicherheit des Teilchenzählens zustande kommt. Bei höheren Teilchenzahlen $(>\sim 80)$ ist ein direktes Auszählen nicht mehr möglich; auf welche Weise man jedoch hier zu brauchbaren Energiewerten gelangen kann, ist in der zitierten Arbeit von $\mathrm{O}$ t t angegeben. In ungünstigen Fällen (geringe Plattenzahl bei hoher Teilchendichte) sind Fehlmessungen um einen Faktor zwei möglich, im allgemeinen beträgt aber der mittlere Fehler nur etwa $20 \%$. Faßt man Schauer gleicher Energie in Gruppen zusammen, so heben sich auch hier die Fehler der Einzelmessungen zum großen Teile heraus.

\section{Ergebnisse}

Eine Aufstellung aller 854 Schauer klassifiziert nach der Energie $E_{\pi^{0}}$ der $\pi^{0}$-Komponente und nach der Zahl $n_{\mathrm{s}}$ der durchdringenden Teilchen bringt Tab. 1. Dabei bedeuten in Anlehnung an die Bristoler Statistiken ${ }^{17,20}$ die Indizes $n$ und $p$ neutrales bzw. geladenes Primärteilchen; ein Fragezeichen heißt, daß die Natur des Primärteilchens unsicher war. Die aufrechten Zahlen gelten für Schauer aus Blei, die kursiven für solche aus Kohle.

\section{A. Geladene und neutrale Primärteilchen}

Aus Tab. 1 läßt sich das Verhältnis der geladenen zu den neutralen Schauerprimären entnehmen; es ist in Tab. 2 gesondert dargestellt in Abhängigkeit von $E_{\pi^{0}}$ und $n_{\mathrm{s}}$. Im Mittel beträgt dieses Verhältnis $p / n=2,3 \pm 0,3$. Für das eine Fünftel der Schauer, deren Primäre aus Richtung der Schneedecke (Abstand 3 bis $4 \mathrm{~m}$ ) kamen, lautet es $p / n=2,7 \pm 0,7$. Andererseits ergab sich aus früheren Messungen in 160 m Höhe mit der gleichen Kammer und mit der

** Die Arbeit ist im Anschluß an die vorliegende in Z. Naturforschg. 9a, 488 [1954] abgedruckt.

${ }^{20}$ R. H. Brown, U. Camerini, P. H. Fowler, H. Heitler, D. T. King u. C. F. Powell, Phil. Mag. 40, 862 [1949].

${ }_{21}$ M. B. Gottlieb, Phys. Rev. 82, 349 [1951].

22 A. Lovati, A. Mura, G. Tagliaferri u. S. Terrani, Nuovo Cim. 9, 946 [1952]. gleichen Zählrohrsteuerung ein $p / n$-Verhältnis $2,5 \pm$ 0,4 . Man mag daraus entnehmen, daß die Vorgeschichte der schauererzeugenden Teilchen in Luft oder ähnlichem leichten Material ohne merklichen Einfluß auf das $p / n$-Verhältnis bleibt. In Tab. 2 deutet sich jedoch eine Abhängigkeit von der Energie an, die auch in Messungen mit Photoplatten 17, 20 zutage tritt. Diese Abhängigkeit mag zum Teil dafür

\begin{tabular}{|c|c|c|c|c|c|c|c|c|c|c|}
\hline $\begin{array}{c}E_{\pi^{0}} \\
(\mathrm{GeV})\end{array}$ & $\begin{array}{l}0,0 \\
0,4\end{array}$ & $\begin{array}{l}0,5 \\
0,9\end{array}$ & $\begin{array}{l}1,0 \\
1,9\end{array}$ & $\begin{array}{l}2,0 \\
2,9\end{array}$ & $\begin{array}{l}3,0 \\
3,9\end{array}$ & \begin{tabular}{|l|}
4,0 \\
4,9
\end{tabular} & \begin{tabular}{|l|}
5,0 \\
9,4
\end{tabular} & $\begin{array}{r}9,5 \\
16,4\end{array}$ & $\begin{array}{l}16,5 \\
29,9\end{array}$ & $\begin{array}{l}30 \\
\infty\end{array}$ \\
\hline$p / n$ & 2,3 & 2,3 & 1,6 & 2,4 & 2,4 & $|2,7|$ & 2,4 & 2,7 & 6 & 8 \\
\hline$n_{\mathrm{S}}$ & 0 u. 1 & 2.u.3 & 4 u. 5 & 6 u. 7 & 8 u. 9 & $10 \mathrm{~b} 1$ & 13 & 13 & & \\
\hline$p / n$ & 3,0 & 2,1 & 1,8 & 2,6 & 2,3 & 2,8 & & 3,0 & & \\
\hline
\end{tabular}

Tab. 2. Das Verhältnis $p / n$ der Zahl der Schauer mit ionisierendem $(p)$ und mit nichtionisierendem $(n)$ Primärteilchen in Abhängigkeit von $E_{\pi^{0}}$ und $n_{\mathrm{S}}$.

verantwortlich sein, daß andere Messungen ${ }^{21-26}$ des $p / n$-Verhältnisses je nach wirksamem Energiebereich recht unterschiedliche Werte zwischen 0,7 und 4,0 ergeben haben.

\section{B) Die Stoßschicht in Kohle}

Vergleicht man die Zahl der Kohleschauer mit der der Bleischauer, so lassen sich Angaben über das Verhältnis der Stoßquerschnitte beider Substanzen machen. Hierbei ist es jedoch nötig, die Schauerhäufigkeiten auf den gleichen Entstehungsort in der Kammer zu beziehen, denn wegen des ortsabhängigen Raumwinkels, unter dem die Zählrohre erscheinen, und wegen der ebenfalls mit dem Ort variierenden Absorberdicken ist die beobachtete Schauerzahl in den einzelnen Platten der Kammer nicht gleich groß. Um daher die Zahl derjenigen Schauer zu finden, die an der Stelle der Kohleplatte in einer Bleischicht erzeugt worden wären, erscheint es am einfachsten, die Schauerhäufigkeiten der ersten und der zweiten Bleiplatte (die der Kohleplatte unmittelbar benachbart sind) zu mitteln. Man hat somit 91 Schauer aus der $5 \mathrm{~cm}$ dicken Kohleschicht (Dichte $1,65 \mathrm{~g} / \mathrm{cm}^{2}$ ) mit 104,5 Schauern (Mittel aus 96 und 113) aus Blei von $1,5 \mathrm{~cm}$ Dicke zu vergleichen. Das Verhältnis der Stoßschichten von Kohle und Blei ergibt sich daraus

${ }^{23}$ W. D. Walker, Phys. Rev. 77, 686 [1950].

${ }^{24}$ K. Sitte, Phys. Rev. 78, 721 [1950].

25 G. Cocconi, V. Cocconi-Tongiorgi u. M. Widgoff, Phys. Rev. 79, 768 [1950].

${ }^{26}$ K. Greisen u. W. D. Walker, Phys. Rev. 90, 915 [1953]. 
zu 0,56. Setzt man voraus, daß in Blei die Schauer mit dem geometrischen Kernquerschnitt erzeugt werden $^{1}$, was einer Stoßschicht von $160 \mathrm{~g} / \mathrm{cm}^{2}$ entspricht, so folgt daraus für Kohle die Stoßschicht zu

$$
\lambda_{\text {Kohle }}=(90 \pm 17) \mathrm{g} / \mathrm{cm}^{2} .
$$

DieserWert paßt zu den Messungen anderer Autoren an Kohle ${ }^{2-8}$, er ist jedoch um das 1,5-fache höher als die einem $A^{2 / 3}$-Gesetz entsprechende Stoßschicht von nur $60 \mathrm{~g} / \mathrm{cm}^{2}$, d. h. der Wirkungsquerschnitt des Kohlekernes beträgt hinsichtlich der Mesonenerzeugung nur $2 / 3$ seines geometrischen Kernquerschnittes.

\section{Die Spektren der $\pi^{0}$-Mesonen und der durchdringenden Schauerteilchen}

In Abb. 2 ist nach den Daten von Tab. 1 das integrale Spektrum der in $\pi^{0}$-Mesonen abgegebenen Energien $E_{\pi^{0}}$ wiedergegeben. Die drei Kurven gelten von unten angefangen für Kohleschauer, für die mittlere Zahl der Schauer in den beiden obersten Bleiplatten und für alle Bleischauer. Wie man sieht, sind die Spektren der Blei- und der Kohleschauer einander ähnlich. Für Bleischauer läßt sich das Spektrum oberhalb etwa $5 \mathrm{GeV}$ durch ein Potenzgesetz der Form

$$
H\left(\geq E_{\pi^{0}}\right)=c_{1} E_{\pi^{0}}^{-\gamma} \quad \text { mit } \quad \gamma=1,5 \pm 0,2
$$

darstellen. Die Messungen an Kohleschauern sind mit dem gleichen Gesetz verträglich, doch dürfte hier die untere Gültigkeitsgrenze sogar etwas tiefer liegen. Daß die Spektren bei niedrigen Energien abflachen, läßt sich leicht durch die selektive Wirkung der Zählrohrsteuerung erklären, der zufolge Schauer kleinerer Energie bzw. zu niedriger Zahl durchdringender Sekundärer mit geringerer Wahrscheinlichkeit aufgenommen wurden. Daher zeigt sich ein entsprechendes Abflachen auch in den Kurven der Abb. 3, der integralen Verteilung der Zahl der durchdringenden Schauerteilchen. Auch hier ist es möglich, die Messungen oberhalb einer gewissen Teilchenzahl ( $n_{\mathrm{s}} \approx 9$ für Blei und $n_{\mathrm{s}} \approx 7$ für Kohle) durch ein Potenzgesetz

$$
H\left(z n_{\mathrm{s}}\right)=c_{2} n_{\mathrm{s}}{ }^{-\delta} \text { mit } \delta=3,8 \pm 0,6
$$

darzustellen.

Die in Abb. 2 und 3 wiedergegebenen Messungen lassen sich mit denen anderer Autoren vergleichen. Unter der Voraussetzung, daß die Energien $E_{\pi^{0}}$ im Mittel proportional zu den Primärenergien $E_{\mathrm{p}}$ der Schauer sind (was weiter unten noch ausführlicher untersucht wird), und in der Annahme, daß die Schwankungen in der Energieaufteilung auf die Sekundären keinen wesentlichen Einfluß auf deren Spektren ausüben, sollte der hier gemessene Exponent $\gamma$ auch für das Spektrum der Schauerprimären

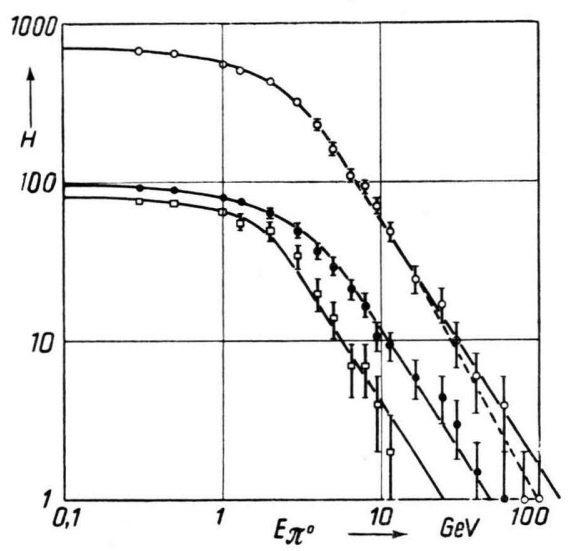

Abb. 2. Das integrale Energiespektrum der in den Schauern enthaltenen Elektronen-Photonenkomponente. $H=$ integrale Schauerhäufigkeit. Die oberste Kurve -०-0-०- gilt für sämtliche Bleischauer, die unterste- $\square$ - $\square-\square$ - für Kohleschauer und die dazwischen-


in der ersten und zweiten Bleiplatte.

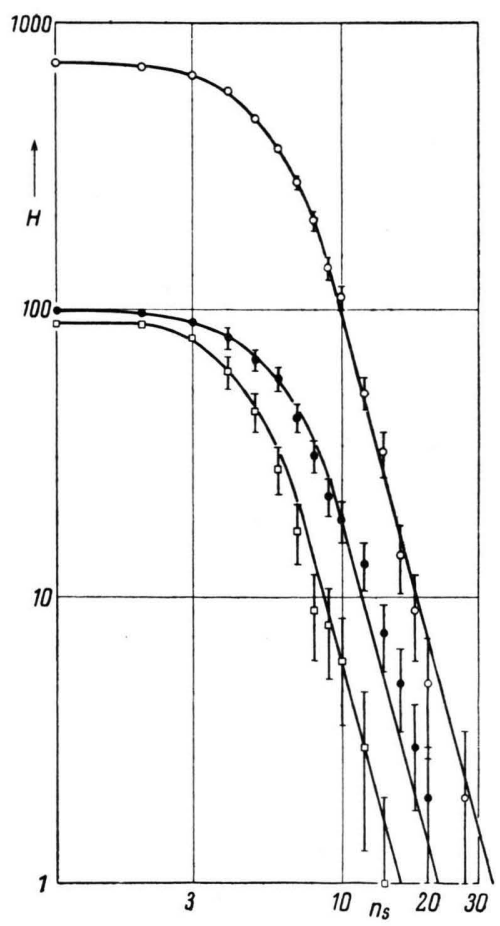

Abb. 3. Die integrale Häufigkeit $H$ der Schauer mit $n_{\mathrm{s}}$ durchdringenden Sekundärteilchen (Multiplizitätsspektrum). Die Reihenfolge der drei Kurven ist die gleiche wie in Abb. 2. 
gültig sein. Es ist deshalb interessant, daß unser $\gamma$-Wert gut zu der Messung paßt, die Mylroi und Wilson ${ }^{27}$ an Protonen (allerdings nur bis $5 \mathrm{GeV}$ ) in Meereshöhe ausgeführt haben. Die Übereinstimmung kann als Bestätigung der angenommenen Proportionalität zwischen $E_{\pi^{0}}$ und $E_{\mathrm{p}}$ angesehen werden.

Auch beim Exponenten $\delta$ ist ein Vergleich mit anderen Messungen möglich. Aus den Multiplizitätsspektren, die Walker et al. ${ }^{8}$ für durchdringende Schauer aus Blei, Eisen und Kohle angegeben haben, läßt sich ein einheitlicher Exponent $\delta$ zu 3,7 entnehmen, der sehr gut zu vorliegender Messung paßt. Auch das Multiplizitätsspektrum der Bristoler Statistik ${ }^{20}$ für $3500 \mathrm{~m}$ Höhe schließt sich für $n_{\mathrm{s}} \geq 8$ mit einem $\delta$-Wert von etwa $3,3 \pm 1,0$ unserer Messung befriedigend an. Bei kleinerem $n \mathrm{~s}$ sinkt jedoch nach dem Bristoler Material $\delta$ leicht ab, was auch in Abb. 3 zu erkennen ist und dort wohl nicht nur auf den Abschneideeffekt der Zählrohre zurückzuführen ist. Auf diese Feinheit wollen wir weiter unten noch zurückkommen.

Es soll nun untersucht werden, welche physikalischen Aussagen aus den in Abb. 2 und 3 wiedergegebenen Messungen und speziell aus der Kenntnis der Exponenten $\gamma$ und $\delta$ zu erhalten sind. Rein formal lassen sich die beiden Gln. (1) und (2) einander gleichsetzen, denn die ausgedrückten Häufigkeitswerte beziehen sich ja auf das gleiche Aufnahmematerial. Man gewinnt somit eine Beziehung zwischen $n_{\mathrm{s}}$ und $E_{\pi^{\circ}}$

$$
n_{\mathrm{s}}=c_{3} E_{\pi^{0}}{ }^{\gamma / \delta} \text { mit } \gamma / \delta=0,4 \pm 0,12,
$$

die nach den Voraussetzungen von Gl. (1) und Gl. (2) zwischen $n_{\mathrm{s}} \approx 9$ und $n_{\mathrm{s}} \approx 25$ gültig sein soll. Die Konstante $c_{3}$ ergibt sich zu

und

$$
\begin{aligned}
& c_{3}=(4,3 \pm 0,2) \mathrm{GeV}^{-1} \text { für Blei } \\
& c_{3}=(4,15 \pm 0,6) \mathrm{GeV}^{-1} \text { für Kohle. }
\end{aligned}
$$

Da beide Werte praktisch übereinstimmen, können wir sie als gleich annehmen und schreiben

$$
n_{\mathrm{s}}=4,3 \cdot E_{\pi^{0}}{ }^{0,4}\left(E_{\pi^{0}} \text { in } \mathrm{GeV}\right),
$$

gültig sowohl für Blei wie für Kohle. Wegen der ziemlich großen Schwankungen, denen die $n_{\mathrm{s}}$ - und $E_{\pi^{\prime}}$-Werte nach Tab. 1 unterworfen sind, darf Gl. $\left(3^{\prime}\right)$ nur im Sinne eines wahrscheinlichsten Zusammenhanges zwischen $n_{\mathrm{s}}$ und $E_{\pi^{0}}$ aufgefaßt werden.

${ }^{27}$ M. G. Mylroi u. J. G. Wilson, Proc. Phys. Soc. Lond. A 64, 404 [1951].
Tab. 1 kann man als Reliefkarte des Zusammenhanges zwischen $n_{\mathrm{S}}$ und $E_{\pi^{0}}$ betrachten mit einem Höhenrücken, der gemäß Gl. $\left(3^{\prime}\right)$ von links oben nach rechts unten verläuft und dabei entsprechend der fortschreitenden Primärenergie ständig an Höhe verliert. Diese Vermutung läßt sich eingehender prüfen, indem

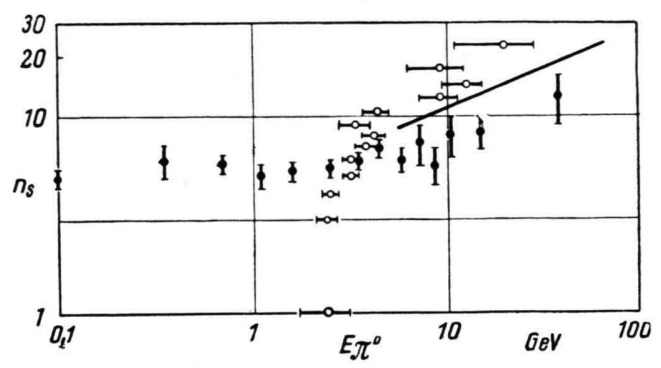

Abb. 4. Die Korrelation zwischen $E_{\pi^{0}}$ und $n_{\mathrm{s}}$ für Bleischauer. Die leeren Kreise stellen die Mittelwerte von $E_{\pi^{0}}$ als Funktion von $n_{\mathrm{s}}$ dar; die ausgefüllten Kreise bezeichnen die Mittelwerte von $n_{\mathrm{s}}$ in Abhängigkeit von $E_{\pi^{0}}$. Als Fehlergrenzen sind die statistischen Fehler angegeben. Die ausgezogene Kurve gilt nach Gl. $\left(3^{\prime}\right)$.

man z. B. für jedes $n_{\mathrm{s}}$ den Mittelwert von $E_{\pi^{0}}$ und für jedes $E_{\pi^{0}}$ den Mittelwert von $n_{\mathrm{S}}$ bildet. Das Ergebnis solcher horizontalen und vertikalen Mittelungen ist aus Abb. 4 ersichtlich. Die zwei Gruppen von Mittelwerten fallen zwar nicht zusammen, doch liegt die Gl. $\left(3^{\prime}\right)$ darstellende Kurve (innerhalb ihres Gültigkeitsbereiches $9<n_{\mathrm{s}}<25$ ) gerade in der Mitte zwischen den beiden Gruppen. Das Auseinanderlaufen der Mittelwerte ist leicht erklärlich: Bei niederen Argumenten ist es bedingt durch den Abschneideeffekt

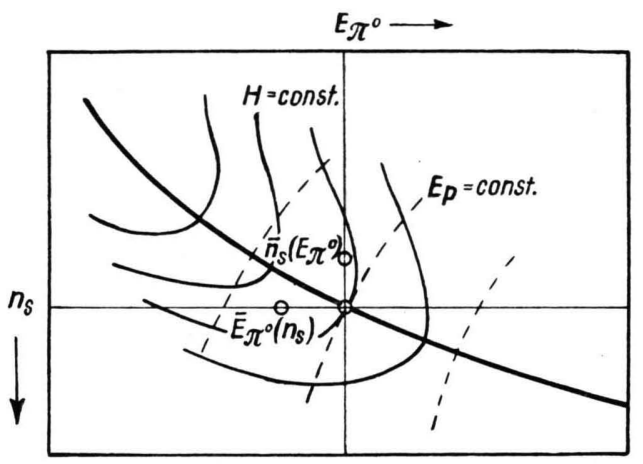

Abb. 5. Schematische Skizze des ,Höhenrückens“, der durch die Schauerhäufigkeiten $H$ von Tab. 1 dargestellt wird.

der Zählrohre und daher hier uninteressant. Jenseits der Abschneidegrenze müßte man, um die Lage des Höhenrückens zu erhalten, über Ereignisse konstanter Primärenergie mitteln, also längs Kurven, die den Höhenrücken senkrecht schneiden (siehe Abb. 5). Tut man es dagegen, wie in Abb. 4 geschehen, längs der Geraden mit konstantem $n_{\mathrm{s}}$ bzw. konstantem $E_{\pi^{0}}$, so erhält man wegen des zu großen Beitrages niedriger Primärenergien zu kleine Mittelwerte. Man ersieht 
jedoch aus Abb. 5, daß der Höhenrücken zwischen den beiden Mittelwertsgruppen verlaufen muß, und gerade dies tut in $\mathrm{Abb} .4$ die ausgezogene Kurve, so daß wir annehmen können, daß Gl. $\left(3^{\prime}\right)$ tatsächlich mit guter Genauigkeit den Höhenrücken, und in diesem Sinne einen wahrscheinlichsten Zusammenhang zwischen $n_{\mathrm{s}}$ und $\boldsymbol{E}_{\pi^{0}}$ darstellt.

Daß bei der Bildung des Mittelwertes $\overline{n_{\mathrm{s}}}$ unter konstant gehaltenem $E_{\pi^{0}}$ die kleinen $n_{\mathrm{s}}$-Werte einen großen Beitrag liefern, läßt sich auch aus Abb. 6 ersehen: hier ist für den Bereich $11,5 \mathrm{GeV} \leq E_{\pi^{0}} \leq \mathbf{2 4 , 4}$

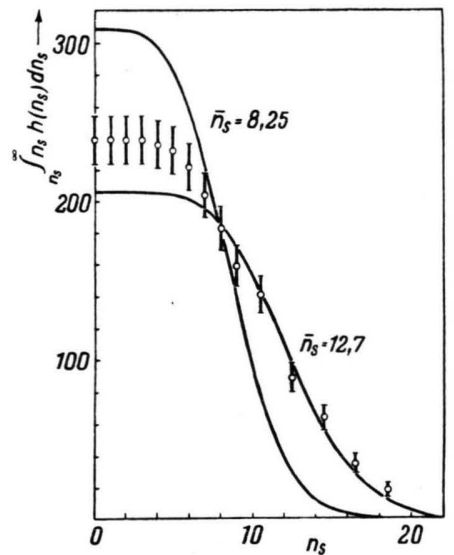

Abb. 6. Die Zahl der durchdringenden Teilchen $n_{\mathrm{s}} \cdot h\left(n_{\mathrm{s}}\right)$ (hierbei bedeutet $h\left(n_{\mathrm{s}}\right)$ die Häufigkeit der Schauer mit $n_{\mathrm{s}}$ Teilchen) als Funktion von $n_{\mathrm{s}}$ in integraler Darstellung für Schauer, deren $\pi^{0}$-Energie zwischen 11,5 und 24,4 GeV liegt. Die beiden Kurven stellen integrale Poisson-Verteilungen dar für die Mittelwerte $\bar{n}_{\mathrm{s}}=8,25$ (bei konstantem $E_{\pi^{0}}$ errechnet) und $\bar{n}_{\mathrm{s}}=1,27$ [nach Gl. $\left.\left(3^{\prime}\right)\right]$.

GeV (Mittelwert 15,0 GeV) die Verteilung der verschiedenen $n_{\mathrm{s}}$ um ihren Mittelwert aufgetragen, und der besseren Statistik wegen ist die integrale Form der Verteilung gewählt. Die ausgezogenen Kurven stellen integrale Poisson-Verteilungen dar, die eine gilt für den bei konstantem $E_{\pi^{0}}$ errechneten Mittelwerte $\overline{n_{\mathrm{S}}}=$ 8,25 und die andere für den nach Gl. $\left(3^{\prime}\right)$ sich ergebenden Wert $\overline{n_{\mathrm{s}}}=12,7$. Man sieht, daß die zweite Kurve oberhalb $\overline{n_{\mathrm{s}}}=7$ recht gut zu den Meßpunkten paßt, daß aber bei kleinen $n_{\mathrm{s}}$-Werten ein deutlicher Überschuß an Schauern zu beobachten ist, der daran schuld ist, daß der (bei konstantem $E_{\pi^{0}}$ ) berechnete Mittelwert $\overline{n_{\mathrm{s}}}=8,25$ so klein ausfällt.

Aus Gl. (3') läßt sich eine Angabe über die mittlere Energie der durchdringenden Schauerteilchen entnehmen. Hierzu setzen wir an, daß die Zahl der gealdenen $\pi$-Mesonen im Mittel gleich der doppelten

28 A. G. Carlson, J. E. Hooper u. D. T. King, Phil. Mag. 41, 701 [1950].

${ }^{29}$ R. R. Daniel, J. H. Davies, J. H. Mulvey u. D. H. Perkins, Phil. Mag. 43, 753 [1952].

${ }^{30}$ G. Salvini u. Y. B. Kim, Phys. Rev. 88, 40 [1952].
Zahl der neutralen ist ${ }^{22,28-30}$, und daß außer den $\pi^{0}$-Mesonen keine weitere Quelle für Elektronenkaskaden existiert. Außerdem wird man annehmen dürfen, daß die Energie (einschließlich Ruhenergie) eines geladenen $\pi$-Mesons im Mittel gleich der Energie eines neutralen ist, d. h. für die Gesamtheit der geladenen $\pi$-Mesonen lautet $E_{\pi_{ \pm}}=2 E_{\pi^{0}} *$. Nicht ganz sicher ist der Energiebetrag, der auf die Protonen unter den Schauerteilchen entfällt. UnterVerwendung des Bristoler Energiespektrums ${ }^{18}$. der Schauerteilchen und in Anbetracht dessen, daß in unserer Messung $\pi^{ \pm}$-Mesonen ab etwa $100 \mathrm{MeV}$ und Protonen ab etwa $400 \mathrm{MeV}$ zu den durchdringenden Teilchen gezählt wurden, gelangt man zu einem Energiebeitrag der Protonen von etwa $0,35 \cdot E_{\pi \pm}$, d. h. für die Energiesumme der durchdringenden Teilchen gilt etwa $E_{\mathrm{s}}=2,7 \cdot E_{\pi^{0}}$, und mit Gl. $\left(3^{\prime}\right)$ erhält man

$$
n_{\mathrm{s}}=2,9 \cdot E_{\mathrm{s}}^{0,4}\left(E_{\mathrm{s}} \text { in } \mathrm{GeV}\right) .
$$

Für die mittlere Energie pro Schauerteilchen ergibt sich daraus

$$
\widetilde{E} \equiv E_{\mathrm{s}} / n_{\mathrm{s}}=0,07 \cdot n_{\mathrm{s}}^{1,5}(\widetilde{E} \text { in } \mathrm{GeV})
$$

unabhängig vom Material (Kohle oder Blei) und gültig für $9<n_{\mathrm{s}}<25$. Während bei niedrigeren $n_{\mathrm{s}}$ -

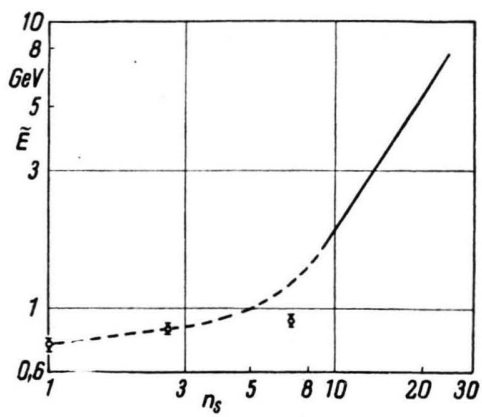

Abb. 7. Die mittlere Energie $\widetilde{E}$ pro durchdringendes Schauerteilchen (bei Mesonen einschließlich Ruhenergie) in Abhängigkeit von $n_{\mathrm{s}}$. Die ausgezogene Kurve ergibt sich aus vorliegender Messung, diePunkte gelten nach Camerini et $\mathrm{al}^{17}$.

Werten $\widetilde{E}$ bekanntlich fast konstant bleibt ${ }^{17}$, steigt es hier mit wachsendem $n_{\mathrm{s}}$ an (vgl.Abb. 7). Der Übergang zu konstantem $\widetilde{E}$ vollzieht sich mit sinkendem $n_{\mathrm{s}}$ offenbar dadurch, daß $\delta$, wie bereits erwähnt,

* Bei höheren Energien müßte man noch den Beitrag der K-Mesonen ${ }^{29}$ berücksichtigen, doch bleibt dieser, wie weiter unten noch gezeigt wird, kleiner als die Fehlergrenze unserer Messung. 
unterhalb $n_{\mathrm{s}} \approx 8$ abnimmt und damit den Exponenten $\gamma / \delta$ in Gl. (3) gegen eins gehen läßt.

In den Abb. 2 und 3 fällt auf, daß die Blei- und die Kohlekurven gegeneinander verschoben sind, auch dann, wenn man sie auf gleiche Absolutintensität normiert, wobei die Normierung nur so viel bedeutet, wie Angleichung der Dicken der Blei- und der Kohleplatten im Verhältnis der Wirkungsquerschnitte (s. o.), so daß die Gesamtzahl der Blei- und der Kohleschauer gerade gleich groß wird. Die verbleibende Verschiebung der Kurven bei größeren Abszissenwerten muß einen physikalischen Grund haben, der durch die unterschiedliche Mesonenproduktion in Kohle und in Blei bedingt ist. Deshalb ist es wichtig, diesen Punkt eingehend zu diskutieren: Man kann z. B. mitW a lker und Mitarbb. ${ }^{8}$ die Verschiebungen als vertikal auffassen und sagen, daß nach Abb. 3 Schauer kleinerer Multiplizität $\left(n_{s} \leq 3\right)$ in Kohle und in Blei etwa gleich häufig entstehen, daß dagegen solche größerer Teilchenzahl in Kohle um etwa einen Faktor 3 seltener werden als in Blei; und ein ähnlicher Schluß ergibt sich für die $\pi^{0}$-Energien nach Abb. 2 . Nun sind aber gleiche $n_{\mathrm{s}}$ - bzw. gleiche $E_{\pi^{0}}$-Werte keine sehr zweckmäßigen Größen für einen direkten Vergleich, denn beide hängen nicht nur von der Primärenergie, sondern auch von der Größe des getroffenen Kerns ab. Man vergleicht also Ereignisse, die von verschiedenen Primärenergien hervorgerufen werden. Weitaus besser ist es deshalb, die Unterschiede der Blei- und der Kohleschauer bei gleicher Primärenergie zu betrachten, und dies ist möglich, wenn man von der horizontalen Verschiebung der Kurven ausgeht. Man überlegt sich leicht, daß die in Abb. 2 und 3 als Ordinate angegebene Schauerhäufigkeit ein vom Material unabhängiges Maß für die Primärenergie ist; denn einmal besteht nach dem bekannten Energiespektrum ein eindeutiger Zusammenhang zwischen der Häufigkeit eines Primärteilchens und seiner Energie. Hat man aber zum anderen die Bleiund die Kohlekurven in dem angegebenen Sinne normiert, so ist auch die Häufigkeit der Schauer unabhängig vom Material ein eindeutiges Maß für die Primärenergie. Die einzige Voraussetzung, die hier zu machen ist, ist die, daß die an Sekundärteilchen abgegebene Energie $E_{\text {sek }}$, welche teils als

* Nach dem oben Gesagten muß man praktisch die gleichen Faktoren erhalten, wenn man die mittleren Teilchenzahlen $\bar{n}_{\mathrm{S}}$ und die mittleren $\pi^{0 \text {-Energien }} \bar{E}_{\pi^{0}}$ aller Blei- und aller Kohleschauer miteinander ver-
$E_{\pi^{0}}$ und teils als Zahl $n_{\mathrm{s}}$ gemessen wird, ihrem Betrage nach für Blei und für Kohle zwar verschieden, aber in ihrem Verhältnis von der Primärenergie unabhängig ist, also ganz links für die Gesamtzahl der Schauer die gleiche ist wir für jeden übrigen Energiewert. (Diese Unabhängigkeit wird durch die offensichtliche Parallelverschiebung der Blei- und der Kohlekurven nahegelegt.) Man liest somit aus den Kurven ab, daß die Teilchenzahl $n_{\mathrm{s}}$ aus Blei um $(30 \pm 15) \%$ höher ist als die aus Kohle, und daß die $\pi^{0}$-Energien sich um einen Faktor $1,9 \pm 0,3$ unterscheiden*.

Es interessiert nun auch, die Primärenergie selbst zu kennen und damit einen Zusammenhang zwischen ihr und der im Mittel erzeugten Zahl der Schauerteilchen zu bekommen. Die Primärenergie ist zwar nicht direkt meßbar, wir können aber versuchen, sie aus der $\pi^{0}$-Energie angenähert zu berechnen: Für die Ableitung der Gl. (4) hatten wir angesetzt, daß die Energie der durchdringenden Schauerteilchen etwa gleich dem 2,7-fachen der $\pi^{0}$ Energie ist. Zählen wir $E_{\pi^{0}}$ selbst hinzu und berücksichtigen wir unter den Nukleonen auch die Neutronen, so ist die Summe der Sekundärenergien für Blei etwa gleich dem 4,7-fachen und für Kohle etwa gleich dem 4,4 -fachen der $\pi^{0}$-Energie, wofür wie in Anbetracht der Unsicherheit der Voraussetzungen einheitlich $E_{\text {sek }}=4,5 \quad E_{\pi^{0}}$ schreiben können. Von der Sekundärenergie $E_{\text {sek }}$ unterscheidet sich die Primärenergie $E_{\mathrm{p}}$ noch um einen Faktor $\varkappa$ der Inelastizität

$$
\varkappa E_{\mathrm{p}}=E_{\mathrm{sek}},
$$

der angibt, ein wie großer Bruchteil der Primärenergie im Mittel an Sekundärteilchen abgegeben wird. Die Absolutwerte von $\varkappa$ sind aus vorliegender Messung nicht zu erhalten, wir können aber schließen, daß der beobachtete Unterschied 1,9 in den $\pi^{0}$-Energien der Blei- und der Kohleschauer als Unterschied der $x$-Faktoren anzusehen ist:

$$
\varkappa_{\mathrm{Pb}} / \varkappa_{\mathrm{C}}=1,9 .
$$

Nun haben Budini und Poiani ${ }^{31}$ aus einer theoretischen Analyse der experimentell bekannten Absorptionslängen der Nukleonenkomponente in Blei und in Kohle $\varkappa_{\mathrm{Pb}}$ zu etwa 0,6 und $\varkappa_{\mathrm{C}} \mathrm{zu} 0,25 \pm 0,1$ bestimmt. Setzen wir demnach $\varkappa_{\mathrm{Pb}}=1 / 2$ und $\varkappa_{\mathrm{C}}=$

gleicht: Es lauten für Blei $\bar{n}_{\mathrm{S}}=6,1 \quad \bar{E}_{\pi^{0}}=4,3 \mathrm{GeV}$ und für Kohle $\bar{n}_{\mathrm{s}}=4,8 \quad \bar{E}_{\pi^{0}}=2,7 \mathrm{GeV}$. Daraus ergeben sich die Verhältnisse $\bar{n}_{\mathrm{sPb}} / \bar{n}_{\mathrm{sc}}=1,3$ und $\bar{E}_{\pi^{0} \mathrm{~Pb}} / \bar{E}_{\pi^{0} \mathrm{c}}=1,6$.

${ }_{31}$ P. Budini u. G. Poiani, Nuovo Cim. 10, 1288 [1953]. 

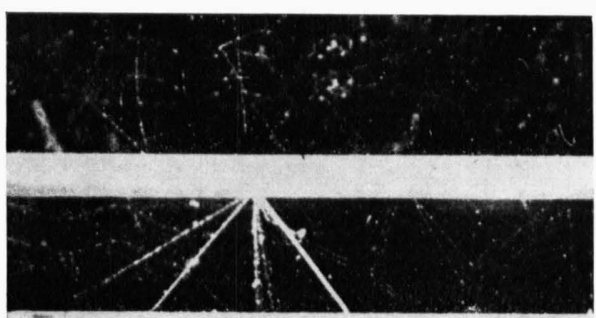

Sinterate
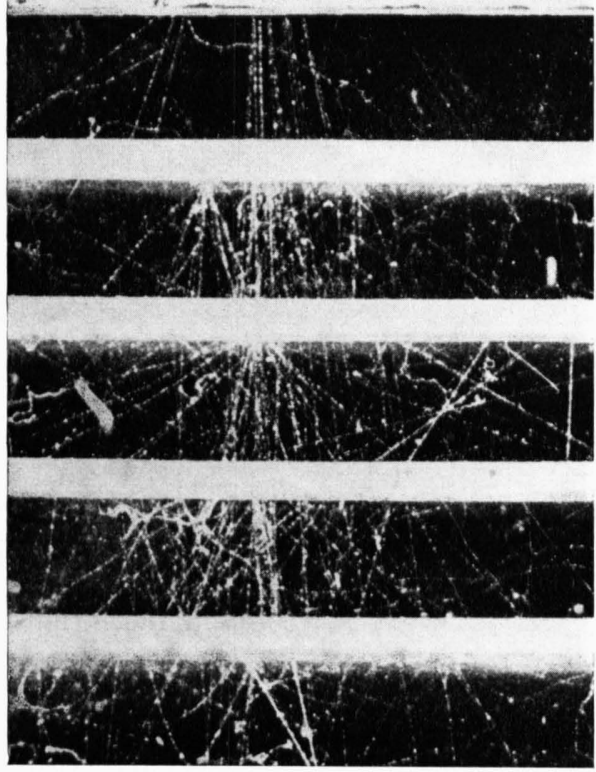

Abb. I. Ein ionisierendes Primärteilchen erzeugt in der obersten Bleiplatte einen Schauer von 6 durchdringenden und 5 stark ionisierenden Teilchen. Einige Elektronenpaare entstehen in der Kohleplatte, doch der überwiegende Teil der Elektronen beginnt erst unter der 2 . Bleiplatte. Man sieht, daß die Elektronenkomponente von nichtionisierenden Teilchen hervorgerufen wird, ihre Energie beträgt $3,5 \mathrm{GeV}$.
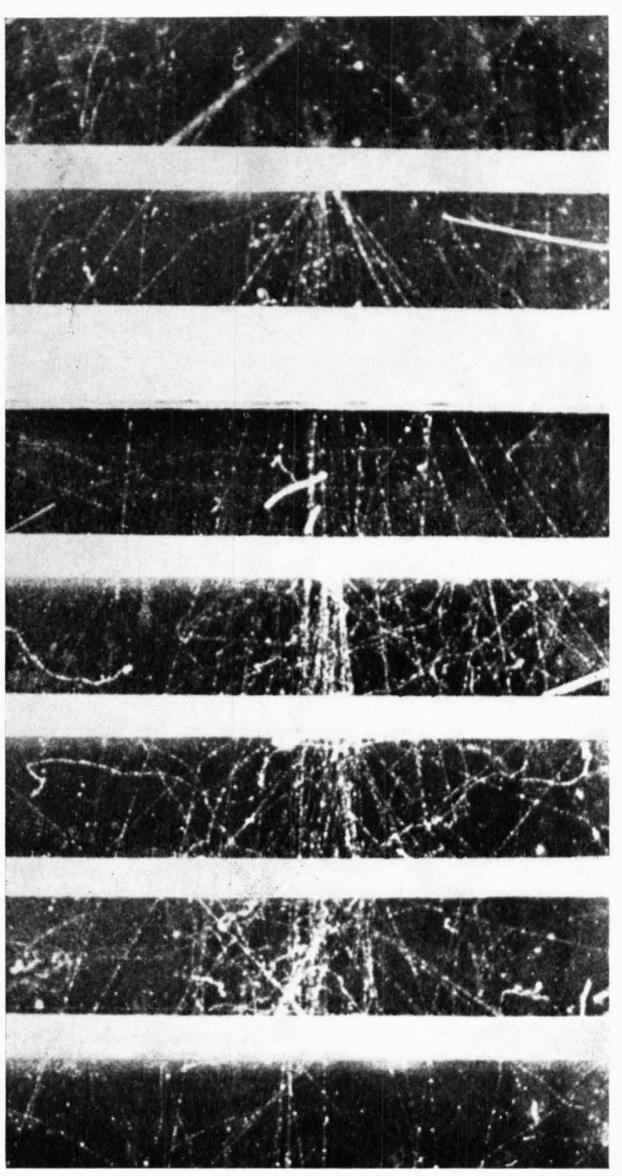

Abb. II. Ein Schauer aus der 1. Bleiplatte, der 8 durchdringende Teilchen enthält und auch inige Elektronen aufweist, die aus der obersten Platte kommen, aber teils in der Kohle und teils in der 2. Bleiplatte stecken bleiben. Die übrige Elektronenkaskade beginnt in der 2 . Bleiplatte und hat eine Energie von $2,1 \mathrm{GeV}$.
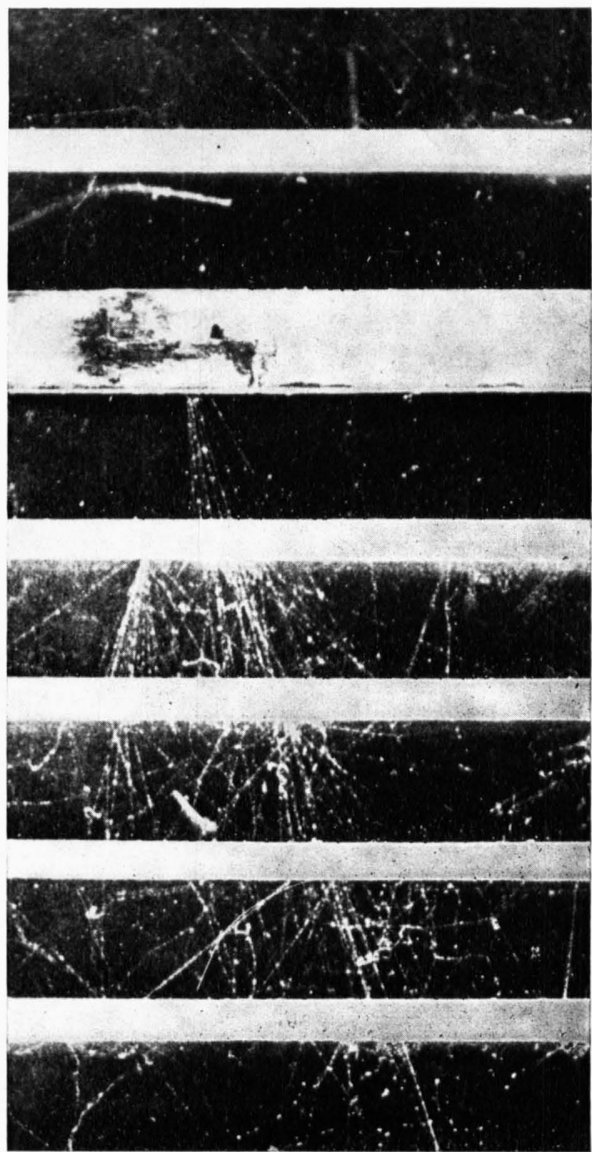

Abb. III. Der Schauer wird in Kohle von einem nichtionisierenden Primärteilchen ausgelöst. Er Energie seiner Elektronenkomponente beträgt $3,0 \mathrm{GeV}$. 
P.O.Schilling und W. Lochte-Holtgreven, Magnetfelder in turbulent strömenden Plasmen (S. 520).

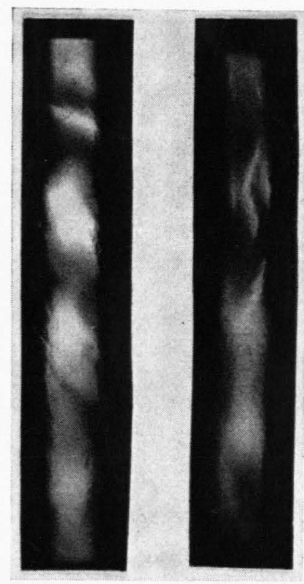

a)

b)

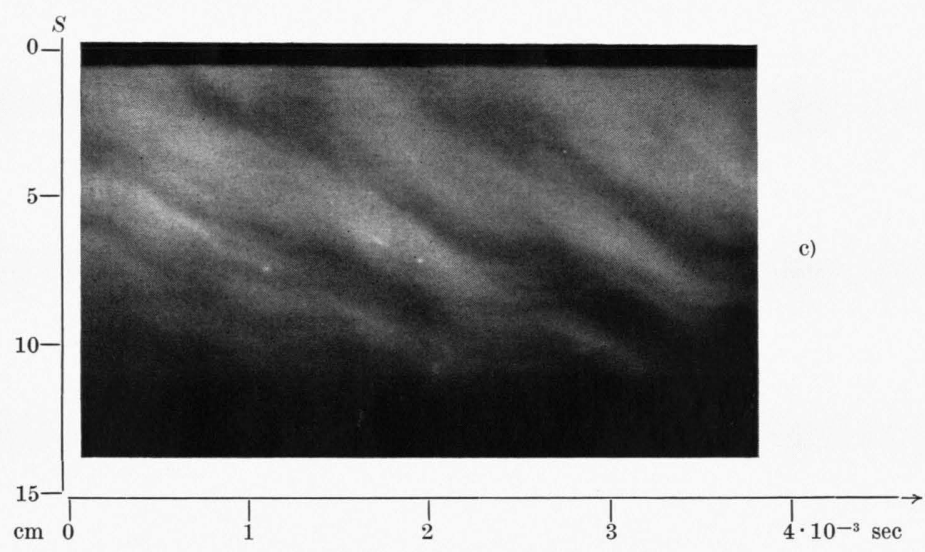

Abb. 3. Brennerflamme. a), b) mit einer Belichtungszeit von $3 \cdot 10^{-4}$ sec, c) im Drehspiegel photographiert. 
$1 / 2 \cdot 1,9$, so erhalten wir für Blei $E_{\mathrm{p}}=\mathbf{2} \cdot \mathbf{4}, 5 \cdot E_{\pi^{0}}$ und für Kohle $E_{\mathrm{p}}=2 \cdot 1,9 \cdot 4,5 \cdot E_{\pi^{0}} * *$. Mit Gl. (3') ergibt sich dann

$$
n_{\mathrm{s}}=1,8 \cdot E_{\mathrm{p}}^{0,4} \text { für Blei }
$$

und

$$
n_{\mathrm{s}}=1,4 \cdot E_{\mathrm{p}}^{0,4} \text { für Kohle }\left(E_{\mathrm{p}} \text { in } \mathrm{GeV}\right) .
$$

Diese beiden Funktionen sind in Abb. 8 als Bleiund als Kohle-Kurven eingezeichnet. Außerdem enthält Abb. 8 ganz links die Messungen der Bristoler Gruppe $^{18}$ an Schauern aus Photoemulsion. Wie man sieht, stellen unsere Kurven eine vernünftige Fortsetzung der Bristoler Daten dar. Außerdem findet man ganz rechts in Abb. 8 drei Punkte für Blei, Messing und Photoemulsion nach Ka plon und Mitarbb. ${ }^{19}$, die aus Messungen mit ,Emulsionskammern" stammen, bei denen die Primärenergie größenordnungsmäßig aus der Winkelbreite der Schauer bestimmt wurde. Die leichte Krümmung unserer Kurven unterhalb $n_{\mathrm{s}} \approx 9$, die sich aus den Gln. (5a) und (5b) noch nicht ergibt, ist dadurch bedingt, daß der Exponent 0,4, wie oben erwähnt, nur für $n_{\mathrm{s}}>9$ gilt; darunter steigt er wegen des absinkenden Exponenten $\delta$ langsam an. Dieses Steilerwerden ist aber in guter Übereinstimmung mit den Bristoler Messungen in Abb. 8. Versucht man außerdem bei höheren Energien den Umstand zu berücksichtigen, daß das 2 : 1-Verhältnis der geladenen Mesonen zu den $\pi^{0}$-Mesonen wegen des Hinzukommens der K-Mesonen nicht mehr gilt, so ergibt sich der gestrichelte Teil unserer Kurven, der unter Verwendung der Bristoler Daten ${ }^{29}$ für 10, 500 und 2000 $\mathrm{GeV}$ gezeichnet wurde. Die Abweichung von der ausgezogenen Kurve ist jedoch nicht sehr groß, sie bleibt noch innerhalb der Fehler, die durch die Ungenauigkeit der Exponenten $\gamma$ und $\delta$ bedingt sind. Um dies zu sehen, ist in Abb. 2 unter der Voraussetzung eines Exponenten 1,5 im Energiespektrum der Primärteilchen die Abweichung von diesem $\gamma$ Wert im Spektrum der $\pi^{0}$-Mesonen gestrichelt eingetragen worden; die Abweichung ist durchaus mit den Meßpunkten verträglich, so daß aus vorliegender Messung keine Aussage mehr zu gewinnen ist, ob der Exponent 0,4 in den Gln. (5a) und (5b) bei sehr hohen Energien etwa nicht mehr gilt.

Außer den experimentellen Kurven enthält Abb. 8 zum Vergleich drei (gestrichelte) theoretische Kur-

\footnotetext{
** Setzt man in diese Beziehungen die mittleren $\pi^{0}$ Energien* ein, so erhält man die mittlere Primärenergie der Schauer zu etwa $50 \mathrm{GeV}$.
}

ven, welche die Zahl der im Nukleon-Nukleon-Stoß erzeugten geladenen Mesonen nach Fermi ${ }^{15}$ und nach Heisenberg ${ }^{16}$ angeben. In der Heisenbergschen Theorie ist als freier Parameter noch der Stoßabstand der beiden Nukleonen enthalten; die untere Kurve stellt deshalb die mittlere und die obere die im Zentralstoß maximal mögliche Mesonenzahl dar.

Abb. 8 veranschaulicht den schon erwähnten Effekt, daß die Zahl der Schauerteilchen aus Blei und aus Kohle sich nur um einen Faktor 1,3 unterscheiden. Daraus darf man wohl schließen, daß die in schweren Elementen möglichen sekundären $\mathrm{Nu}$ -

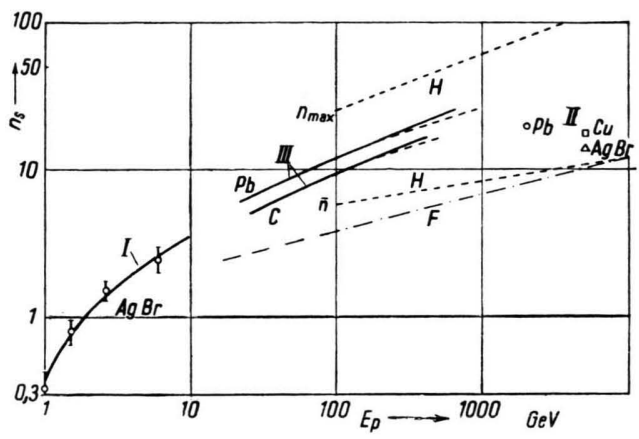

Abb. 8. Die Abhängigkeit der Teilchenzahl $n_{\mathrm{s}}$ von der Primärenergie $E \mathrm{p}$ : links (I) nach Messungen der Bristol-Gruppe ${ }^{18}$ an Photoemulsion, ganz rechts (II) nach Messungen von Kaplon et al. ${ }^{19}$ an Blei $\bigcirc$, Messing $\square$ und Photoemulsion $\triangle$, und in der Mitte (III) der aus vorliegender Messung berechnete Verlauf von $n_{\mathrm{s}}$ für Blei $(\mathrm{Pb})$ und für Kohle $(\mathrm{C})$. Die gestrichelten Kurven gelten für die Zahl der im Nukleon-Nukleonstoß erzeugten geladenen Mesonen nach den Theorien von Fermi $^{15}(\mathrm{~F})$ und Heisenberg ${ }^{16}(\mathrm{H})$.

kleonenstöße nur wenig zur Erzeugung höherer Teilchenzahlen beitragen. Zudem ist nach Gl. (4) die mittlere Energie der Schauerteilchen aus Blei und aus Kohle innerhalb der Meßgenauigkeit gleich groß; es tritt also keine wesentliche Aufsplitterung der Energien durch Sekundärstöße ein. Andererseits verliert aber das Primärteilchen in schweren Kernen einen höheren Energiebetrag als in leichten (Faktor 1,9 zwischen Blei und Kohle), was man als Argument für das Vorhandensein von Sekundärstößen auffassen kann. Man mag diesen Unterschied aber auch so diskutieren, daß das Primärteilchen beim Auftreffen auf einen größeren Kern mit höherer Wahrscheinlichkeit eines der Nukleonen relativ zentral anstößt und dabei sowohl eine höhere Energie verliert als auch eine größere Mesonenzahl erzeugt. Die im Zentralstoß zweier Nukleonen nach $\mathrm{He}$ is en berg maximal mögliche Mesonenzahl reicht 
jedenfalls aus, um die beobachteten Teilchenzahlen zu erklären. Wenn man also annimmt, daß Sekundärstöße für die Mesonenerzeugung von geringem Einfluß sind, so muß die freie Weglänge für einen energiereicheren Stoß im Kern größer als der Nukleonenabstand sein. Damit vereinbar wäre die Beobachtung, daß leichte Kerne, was die Mesonenerzeugung anbetrifft, etwas transparent erscheinen $^{32}$ (siehe Abschnitt B).

\section{Stark ionisierende Teilchen}

Neben den durchdringenden Teilchen und den Elektronenkaskaden wurden in vorliegender Messung auch die stark ionisierenden Teilchen gezählt, und es wurde nach Beziehungen zwischen der Zahl $N_{\mathrm{h}}$ dieser Teilchen und $n_{\mathrm{s}}$ bzw. $E_{\pi^{0}}$ gesucht. Als stark ionisierend wurden dabei nur diejenigen Teilchen betrachtet, deren Ionisation mehr als das 3bis 4 -fache der Minimumsionisation betrug. Die be-

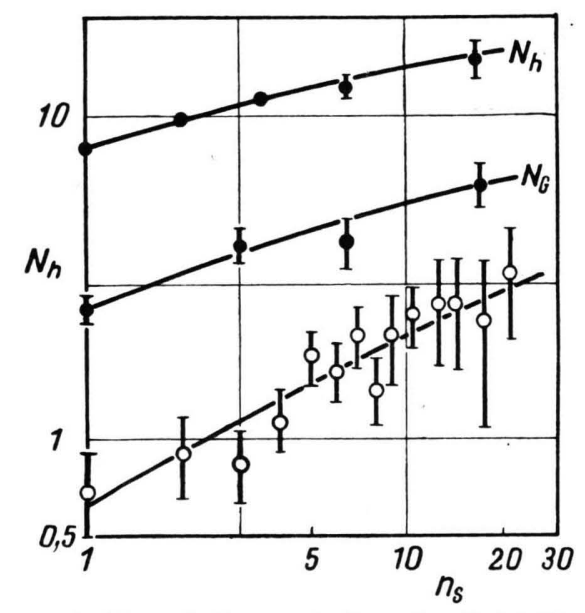

Abb. 9. Die Korrelation zwischen der Zahl $N_{\mathrm{h}}$ der pro Bleischauer emittierten stark ionisierenden Teilchen und der Zahl $n_{\mathrm{s}}$ der durchdringenden Teilchen. Die

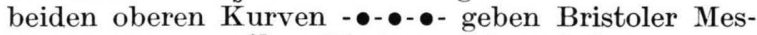
sungen $^{17}$ an Photoemulsion wieder.

obachtete Teilchenzahl hängt natürlich von der Dicke der verwendeten Kohle- und Bleiplatten ab und ist in ihrem Absolutwert nicht ohne weiteres mit anderen Messungen, z. B. mit denen in Photoplatten vergleichbar. Wir können aber in unserem Fall die relativen Zahlen $N_{\mathrm{h}}$ aus Kohle und aus Blei recht gut vergleichen, da die Dicken der Platten $\left(8,3 \mathrm{~g} / \mathrm{cm}^{2}\right.$ Kohle und $17 \mathrm{~g} / \mathrm{cm}^{2}$ Blei $)$ ziemlich genau

${ }^{32}$ D. Froman, J. Kenney u. V. H. Regener, Phys. Rev. 91, 707 [1953]. im Verhältnis der relativen Bremsvermögen von Kohle und von Blei stehen. (Nach den bekannten Reichweitekurven ${ }^{1,33}$ vermag ein Proton oder Meson mit einer Reichweite von $17 \mathrm{~g} / \mathrm{cm}^{2}$ Blei in Kohle gerade rund $8,5 \mathrm{~g} / \mathrm{cm}^{2}$ zurückzulegen.)

Insgesamt wurden 1179 stark ionisierende Teilchen in Schauern aus Blei und 28 in Schauern aus Kohle gezählt, damit beträgt die mittlere Zahl $N_{\mathrm{h}}$ pro Bleischauer $1,54 \pm 0,1$ und pro Kohleschauer nur $0,31 \pm 0,1$, d. h. die Häufigkeiten der stark ionisierenden Teilchen unterscheiden sich um einen Faktor 5. Von den stark ionisierenden Teilchen wurden aus Blei 355 und aus Kohle 4 in den oberen

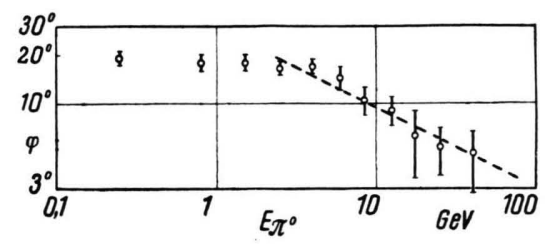

Abb. 10. Die Zahl $N_{\mathrm{h}}$ der pro Bleischauer emittierten stark ionisierenden Teilchen in Abhängigkeit von der Energie $E_{\pi^{0}}$.

Halbraum (entgegengesetzt der Einfallsrichtung des Primärteilchens) emittiert. Wie man aus Photoplattenmessungen $^{17}$ weiß, ist $N_{\mathrm{h}}$ nicht konstant, sondern zeigt einen leichten Anstieg mit der Teilchenzahl $n_{\mathrm{s}}$ des Schauers. Eine derartige Variation ergibt sich nach Abb. 9 auch aus vorliegender Messung. Abgesehen von dem bereits erwähnten Häufigkeitsfaktor paßt unsere Messung recht gut zu der Bristoler. In Abb. 10 ist ferner $N_{\mathrm{h}}$ gegen die Energie der $\pi^{\mathbf{0}}$-Mesonenkomponente aufgetragen, es ist keine merkliche Korrelation zwischen beiden GröBen erkennbar.

\section{E. Die Winkelbreite der Schauer}

Um ein Maß für die Schauerbreite zu gewinnen, wurde für jeden einzelnen der projizierte Winkel $\varphi$ gemessen, innerhalb dessen sich die Hälfte aller durchdringenden Teilchen befindet. In Anbetracht der relativ großen Schwankungen von $\varphi$ wurde es für ausreichend betrachtet, den projizierten Winkel unter dem Stereoskop durch Auflegen einer einfachen Schablone auf die Filme mit einer Genauigkeit von rund 20 bis $30 \%$ zu bestimmen.

${ }^{33}$ H. A. Bethe, AEC-Bericht, BIVTL-T-7 (1949). 
Wie Tab. 3 zeigt, ergibt sich eine deutliche Abhängigkeit der mittleren Winkelbreite von der an der Schauerentstehung beteiligten Nukleonenzahl.

Die Abhängigkeit der Schauerbreite $\varphi$ (der Bleischauer) von $n_{\mathrm{s}}$ und von $E_{\pi^{0}}$ geht aus den folgenden Abb. 11 und 12 hervor. Man sieht, daß mit steigender $\pi^{0}$-Energie die Schauer enger werden, wie man

\begin{tabular}{|c|c|c|}
\hline Material & $N_{\mathrm{h}}$ & $\varphi$ in Grad \\
\hline Kohle & 0 & $10,2 \pm 1,3$ \\
,, & $>0$ & $13,7 \pm 2,8$ \\
\hline Blei & 0 & $16,1 \pm 0,9$ \\
,, & 1 bis 3 & $16,9 \pm 1,0$ \\
, & $>3$ & $23,1 \pm 2,2$ \\
\hline
\end{tabular}

Tab. 3. Die projizierte Winkelbreite $\varphi$ der Kohle- und Bleischauer in Abhängigkeit von der Zahl $N_{\mathrm{h}}$ der stark ionisierenden Teilchen. Die Fehlergrenzen stellen die aus der Zahl der Schauer berechneten statistischen Fehler dar.

es erwartet, wenn die $\pi^{0}$-Energie mit der Primärenergie der Schauer anwächst. Bei nicht zu niedrigen Energien sind die Meßpunkte mit einem Gesetz $\operatorname{tg} \varphi \sim E^{-0,5}$ verträglich, welches für die Mesonenerzeugung im Nukleon-Nukleon-Stoß etwa gelten sollte ${ }^{34}$. Unterhalb $E_{\pi^{0}} \approx 3 \mathrm{GeV}$ ändert sich die Winkelbreite offenbar nicht, dort dürfte wegen des Abschneideeffektes der Zählrohre die $\pi^{0}$-Energie kein gutes Maß mehr für die Primärenergie sein.

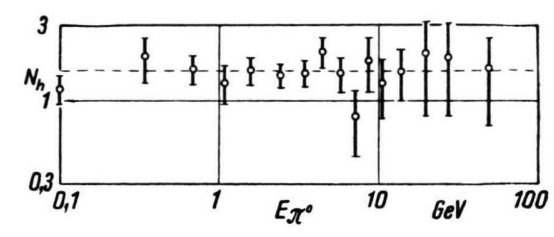

Abb. 11. Der mittlere projizierte Winkel $\varphi$, der die Hälfte aller durchdringenden Schauerteilchen aus Blei umfaßt, in Abhängigkeit von $E_{\pi^{0}}$. Die gestrichelte Kurve stellt eine Beziehung $\varphi \sim E_{\pi^{0}-1 / 2}$ dar.

Einen etwas anderen Verlauf zeigt nach Abb. 12 die Schauerbreite als Funktion von $n_{\mathrm{s}}$. Bei großen Teilchenzahlen $n_{\mathrm{s}}$ mögen die Schauer wohl in der erwarteten Weise gemäß $\operatorname{tg} \varphi \sim E_{\bar{\pi}^{0}} 0,5 \sim n_{\mathrm{S}}^{-1,25}$ enger werden; offensichtlich ist aber noch ein zusätzlicher Effekt wirksam, der bei kleinen $n_{\mathrm{s}}$ zunächst die Schauerbreite anwachsen läßt. Die Erklärung dieses Verlaufes ist nicht ganz einfach, da bei kleinen $n_{\mathrm{s}}$ schon die Abschneidewirkung der Zählrohre mit-

${ }^{34}$ U. Camerini, J. H. Davies, C. Franzinetti, W. O. Lock, D. H. Perkins u. G. Yekutieli, Phil. Mag. 42, 1261 [1951]. spielt. Andererseits deutet sich ein ähnlicher Verlauf auch in den Bristoler Photoplattenmessungen ${ }^{17}$ an. Man mag deshalb vermuten, daß es sich hier um einen Einfluß von Schwankungserscheinungen handelt derart, daß im Falle eines zufälligen Anwachsens der Zahl der Mesonen deren mittlere Energie niedriger wird, was eine stärkere Streuung der Mesonen im Atomkern zur Folge hat.

\section{Zusammenfassung}

Die Ergebnisse der vorliegenden Messung können in folgender Weise zusammengefaßt und gedeutet werden:

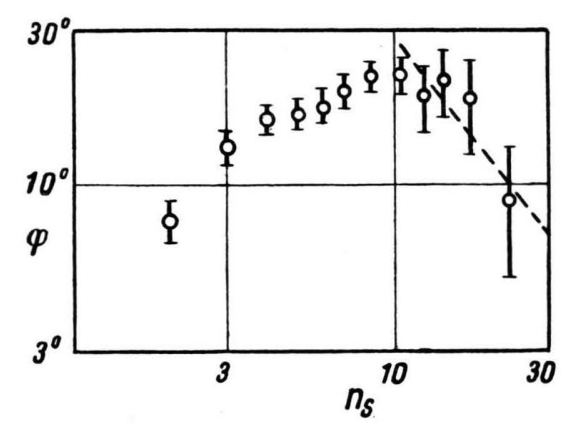

Abb. 12. Der Winkel $\varphi$ in Abhängigkeit von $n_{\mathrm{s}}$. Die gestrichelte Kurve gibt die Beziehung $\varphi \sim n_{\mathbf{S}}{ }^{-1,25}$ wieder.

Aus den Energie- und Multiplizitätsspektren der Blei- und der Kohleschauer lassen sich Aussagen über den Gang der Zahl der durchdringenden Teilchen mit der Primärenergie der Schauer entnehmen. Es zeigt sich, daß $n_{s}$ (im Bereich $9<n_{\mathrm{s}}<25$ ) im Mittel mit der 0,4. Potenz der Primärenergie $E_{\mathrm{p}}$ anwächst, also meßbar steiler als mit der bisher oft angenommenen 4 . Wurzel aus $E_{\mathrm{p}}$. Mit abnehmen$\operatorname{dem} n_{\mathrm{s}}(<9)$ steigt der Exponent sogar über 0,4 an. Diese Aussage gilt sowohl für Blei- wie für Kohleschauer.

Die Schauer aus Blei und aus Kohle unterscheiden sich in ihren $\pi^{0}$-Energien um etwa einen Faktor 2 und in der Zahl $n_{\mathrm{s}}$ der durchdringenden Teilchen um $30 \%$. Der geringe Unterschied in $n_{\mathrm{s}}$ kann wohl nur unter der Voraussetzung einer multiplen Mesonenerzeugung verstanden werden. Es ist sogar möglich, daß die 30 -proz. Zunahme von $n_{\mathrm{s}}$ nur zum Teil auf Kaskadenbildung im Bleikern zurückzuführen ist, und daß auch der Umstand mitspielt, daß in dem viele Nukleonen enthaltenden Bleikern die Wahrscheinlichkeit für einen zentraleren $\mathrm{Zu}$ sammenstoß mit einem Nukleon größer ist als im 
Kohlekern; im zentraleren Stoß könnte sowohl eine höhere Energie übertragen als auch eine größere Mesonenzahl erzeugt werden.

Sehr deutlich machen sich die Sekundärstöße der durchdringenden Schauerteilchen in den energieärmeren Prozessen bemerkbar: Aus Blei werden im Mittel 5-mal mehr stark ionisierende Teilchen emittiert als aus Kohle; und offensichtlich infolge dieser elastischen Stöße verbreitert sich die Garbe der durchdringenden Schauerteilchen aus Blei um fast das Doppelte gegenüber der aus Kohle.

Wenn die Aussage über den geringen Beitrag der Sekundärstöße zur Vermehrung der Schauerteilchenzahl richtig ist, so folgt daraus, daß die Kernmaterie hinsichtlich der Mesonenerzeugung etwas transparent sein muß. In Einklang damit steht der niedrige Wirkungsquerschnitt des Kohlekernes.

Herrn Prof. Dr. W. Heisenberg darf ich für anregende Diskussionen und für seine wohlwollende Förderung der Arbeit danken. Die Messungen auf der Zugspitze wären nicht möglich gewesen ohne die Initiative von Herrn Prof. Dr. K. Wirtz und ohne das Entgegenkommen der Deutschen Bundespost, die uns einen ihrer Räume zur Verfügung gestellt hat. Bei der Einrichtung des Raumes und bei der Vorbereitung für die Aufstellung der Nebelkammer war die Mithilfe der Herren K. Geismann, A. Kaehs und M. Pöschel von großem Wert. Ganz besonders möchte ich auch Herrn K. Böttcher danken, der bei der Bedienung der Nebelkammer mitwirkte und nicht nur die angenehme, sondern auch manche mühsame Situation des Berglebens auf sich nahm.

\title{
Numerische Rechnungen zur Kaskadentheorie Energiebestimmung von $\pi^{0}$-Mesonen
}

\author{
Von KarL OTT \\ Aus dem Max-Planck-Institut für Physik, Göttingen \\ (Z. Naturforschg. 9a, 488-494 [1954]; eingegangen am 10. April 1954)
}

\begin{abstract}
Die Anzahl der Elektronen einer Elektronen-Photonenkaskade in Blei wird als Funktion des Abstands vom Schauerursprung für einige Primärenergien $E_{0}(0,3$ bis $300 \mathrm{GeV})$ numerisch berechnet und zwar für den Fall eines primären Photons bzw. Elektrons der Energie $E_{0}$ und für ein primäres Spektrum von Photonen, wie es aus den beim Stoß zweier Nukleonen erzeugten $\pi^{0}$-Mesonen durch $\pi^{0}-2 \gamma$-Zerfall entsteht. Die zugrunde gelegte Theorie der Elektronen-Photonenkaskade wurde in einer früheren Arbeit ${ }^{1}$ dargestellt.

Die Ergebnisse über die Elektronenverteilung in durchdringenden Schauern gestatten z. B. aus Nebelkammeraufnahmen ${ }^{2}$ solcher Schauer den Energieinhalt der dabei erzeugten $\pi^{0}$-Mesonen zu bestimmen.

Außerdem wurde die von einem Photonenspektrum vom Bremsstrahltyp ausgelöste Elektronenkaskade berechnet und mit den Messungen von Blocker, Kenney und Panof sky ${ }^{3}$ verglichen.

Zum Schluß wurde noch die Möglichkeit zur Energiemessung diskutiert, wenn die Teilchenzahl im Schauerkern so groß ist, daß sie nicht mehr ausgezählt werden kann.
\end{abstract}

$\mathrm{B}$ ei der theoretischen Behandlung der ElektronenPhotonenkaskade wurden ursprünglich für die die Kaskade auslösenden Prozesse, Bremsstrahlung und Paarerzeugung, die Wirkungsquerschnitte im Grenzfall hoher Energie (bei vollständiger Abschirmung) benutzt (ausführliche Literaturhinweise siehe 1. c. ${ }^{1}$ ). In diesem Grenzfall ist z. B. der Absorptionskoeffizient der $\gamma$-Quanten unabhängig von der Energie $\left(\approx^{7} / 9\right)$. Kommen jedoch die Kaskadenteilchen nach Aufsplitterung der Anfangsenergie in den Energiebereich unterhalb etwa $100 \mathrm{MeV}$, dann wird

\footnotetext{
${ }^{1}$ Vorträge über kosmische Strahlung, Herausgeb. W. Heisenberg, Springer 1953 (woraus der größte Teil der Bezeichnungen übernommen wurde).
}

ihre Absorption schwächer; die der $\gamma$-Quanten nimmt aber bei noch kleineren Energien infolge des Compton-Effekts wieder zu. In einer früheren Arbeit ${ }^{1}$ wurden diese Effekte in erster Näherung berücksichtigt.

\section{Die Einteilchenkaskade}

Tritt ein Photon der Energie $E_{0}$ in Materie ein, so löst es bei hinreichend großem $E_{0}$ eine Kaskade aus, die sich im wesentlichen in der Impulsrichtung des Primärteilchens ausbreitet. Die Materialdicke $x$

${ }^{2}$ M. Deutschmann, Z. Naturforschg. 9 a, 477 [1954].

${ }^{3}$ W. Blocker, R. Kenney u. W. Pan of sky, Phys. Rev. 79, 419 [1950]. 